Interpretation of History", aptly illustratos the possibilitios in this direction and also has some implications for the writing of industrial and scientific history that the scientist or technologist might ponder. Mr. D. D. Haslam, when discussing on September 27 the problems of library staffing, covered university and Government libraries as well as those of commerce and industry, the technical colleges and the public libraries, and presented a balanced picture of trends and difficulcies and their implications which is of wide interest. The planning of central libraries was considered by Mr. F. M. Gardner on September 25 in a paper of interest to industry and commerce as well as to public libraries, while on September 26 Mr. P. H. Sewell examined generally presentday thought on library co-operation though without considering the technical implications of the Roberts report. Miss C. Jones, on September 27, under the title "From Book-Box to Telex: Mechanization for County Libraries", discussed some of the developments and opportunities which mechanization had brought to the county library service. Other papers presented at the conference were concorned with libraries and youth. children's reading and aspects of library service in hospitals.

\title{
FORCES IN COLLOIDAL SYSTEMS
}

$\mathrm{A}$ $\mathrm{N}$ informal discussion of the Faraday Society on the nature of forces in colloidal systems was held in the Chemistry Department of the University of Nottingham during September 20-21, by kind permission of the Vice-Chancellor. The meeting was attended by 108 people, 69 of whom came from industrial organizations and 39 from educational institutions.

Colloidal systems are very diverse in character, and the early research on colloids was essentially based on phenomenology. It has therefore become increasingly important, in order to understand systematically the behaviour of such a wide range of systems, to obtain a deeper understanding of the fundamental forces which act between particles, or molecules, of colloidal dimensions. The forces have the same origin as those acting between simple ions and molecules but in colloidal systems the situation becomes more complicated since the 'ion' may consist of a particle with a number of charges on the surface, for example, a micro-crystal of silver iodide, or an assembly of soap ions as in a micelle. Both repulsive and attractive forces have to be averaged over a surface, and in recent years theoretical work has been devoted to obtaining equations which enable the variation of the force to be predicted over distances of the order of those separating colloid particles. The equations require experimental verification of their validity, and in recent years work on the stability of dispersions, on the structure of soap micelles, and on the stability and structure of thin soap films has yielded some measure of the relative contribution of electrical, attractive and solvation forces. This trend was clearly illustrated during the course of the discussion.

On September 20, the afternoon session was entitled "Stability of Dispersions in Aqueous and Non-aqueous Media". Aftor an address of welcome by Prof. D. D. Eley, the chair was taken by Sir Eric Rideal, who, in his introductory remarks, stressed the importance of colloids as a field of study and the need for deeper understanding of the fundamentals of the subject. A great deal of work was still required to elucidate the part played by the solvent both in aqueous and non-aqueous media.

The first paper, by Dr. J. A. Kitchener and Mr. F. Z. Saleeb (Imperial College of Science and Technology), reported work on the adsorption of 'Aerosol $O T$ ' by a graphitized carbon black in aqueous media. The adsorption isothorms showed saturation adsorption corresponding to a close-packed, vertically oriented, monolayer of 'Aerosol $O T$ ' ions having their sulphon- ate hoad groups exposed to the liquid. Comple mentary measurements of zeta potential, surface conductance and the negative adsorption of co-ions were also made. Results suggested that the majority of the counter-ions were located in the Stern plane and that the plane of shear in the electrokinetic experiments was close to the plane of the head groups of the adsorbed 'Aerosol $O T$ ' ions.

Mr. P. Dawson and Dr. D. A. Haydon (University of Cambridge) presented work on the adsorption of a series of $n$-alkyl carboxylic acids by titanium dioxide suspensions in benzene. The dispersions used were of small particle size (radius c. $25 \AA$ ) and it was found that the stability of the dispersion increased as the length of the alkyl chain was increased. It was suggested, therefore, that stabilization did not occur until the chains were sufficiently long for steric hindrance to occur between the adsorbed layers. An exception was found in the case of acetic acid, which also appeared to stabilize the system; it seemed improbable, however, that in this case stabilization could arise from steric hindrance.

An account of adsorption on to 'Graphon', from binary liquid mixtures of monoalkyl benzenes and $n$-heptane, was given by Dr. G. D. Parfitt and Mr. E. Willis (University of Nottingham). The apparent isothermis changed in nature as the length of the alkyl side-chain increased, indicating an increasing preferential adsorption of the alkyl benzene. The adsorption isotherms calculated for the individual components suggested a saturation monomolecular layer of alkyl benzene with the molecules lying flat on the surface. Complementary measurements of stability were made on dilute dispersions of 'Graphon', and it was found that the stability increased, with the proportion of alkyl benzene in the mixture and the length of the alkyl chain. The van der Waals attraction constant calculated for this system, with due allowance for the thickness of the adsorbed layer. appeared to be too large to account for the stability observed experimentally.

Drs. K. G. Mathai and R. H. Ottewill (University of Cambridge) presented the results of work on the stabilization of aqueous inorganic dispersions by non-ionic detergents. The rate of flocculation of sols by bivalent and trivalent ions was measured in the presence of various concentrations of non-ionic detergents. The latter were polyoxyethylene glycol monoalkyl others, the alkyl chain length of which was varied from eight carbon atoms to sixteen; the length of the polyoxyethylene glycol chain was maintained constant at six units. As the concentra- 
tion of non-ionic detergent was increased the concentration of inorganic salt required to produce flocculation also increased. Above the critical micelle concentration of the detergent, however, a large increase in stability of the sol occurred. Below the critical micelle concentration the increase in stability was attributed to the increase in hydration of the sol particles caused by adsorption of a thin layer of detergent molecules. The large increase in stability observed above the critical micelle concentration was considered to be due to the fact that the adsorbed molecules prevented the particles from approaching sufficiently closely to enter the primary minimum.

On September 21, the session was entitled "Micellization", and the chair was taken by Prof. D. D. Eley. The first paper in this session, by Dr. A. S. C. Lawrence and Messrs. R. Aveyard and J. T. Pearson (University of Sheffield), presented the results of studies on micellization using calorimetry. Results were presented for micellization and co-micellization (the case of a polar micelle incorporating amphiphilic molecules) and also for the effects of salt concentration on these phenomena. The heats of solution of a series of $n$-alcohols in water and in soap-water mixtures were discussed in terms of the heats of hydration of the hydrocarbon chain and the polar or ionic head group. The contribution to the energy terms due to increased unbinding of counter-ions in the co-micellization process was discussed, and the calorimetric measurements were supported by measurements of conductance, $p \mathrm{H}$ and sodium ion activity in these systems; the latter measurements involved the use of a sodium responsive glass electrode.

Mr. J. Clifford and Dr. B. A. Pethica (Unilever, Port Sunlight) reported the results of measurements on the self-diffusion of micelles of sodium dodecyl sulphate (dye labelled) and on the self-diffusion of sodium ions (radio-tracer labelled) in the presence of these micelles. The mechanism of binding counterions to the micelle by means of electrostatic forces was discussed. The results of some preliminary studies on micelles using nuclear magnetic resonance were also presented. A change in spin lattice relaxation time of the water protons was found in the presence of the micelles, suggesting that water had been immobilized near the micelle surface. The spin lattice relaxation time of the protons in the $\mathrm{CH}_{2}$ groups in the interior of the micelles was approximately ten times shorter than the relaxation time of $\mathrm{CH}_{2}$ groups in liquid hydrocarbons. This suggested that the alkyl chains in the interior of the micelle were under compression.

The structure and stability of black foam films were discussed by Dr. J. F. Goodman (Procter and Gamble, Ltd., Newcastle upon Tyne). Experiments were described in which simultaneous measurements were made of the total film thickness, using an optical method, and of the water content using infrared spectroscopy. The detergent content of the film was determined by radio-tracer experiments. It was shown that the black film (50-100 $\AA$ thick) has a simple lamellar structure in which two monolayers of surface-active molecules are spread on a thin aqueous core. In mixed cationic-anionic surfaceactive systems it was found that the ratio of anionic to cationic material in the film was always $1: 1$, irrespective of the ratio in the bulk liquid. Under these conditions it appeared unlikely that the film could be stabilized by electrostatic repulsion between opposing monolayers of soap ions. The factors influencing the stability of the thin films were discussed in terms of the evaporative equilibrium of the volatile film component. It was suggested that the stability of a black film could be maintained by an excess of solute, relative to the bulk solution, in the aqueous film core.

Prof. B. Stuke (University of Munich, Germany) discussed the thermodynamics of micellar systems. In the first part of the paper a discussion was given of the respective merits of using the law of mass action to describe micellization and of considering the micelle as a separate phase. It was concluded that thermodynamically the law of mass action gives the sound approach, the phase approach being only an approximation. Starting from a model for the process of micelle formation at interfaces the second part of the paper was concerned with the influence of micelles on the stabilization of thin films.

R. H. OTTEWILL

G. D. ParfitT

\section{GEOLOGY AND GEOPHYSICS IN THE U.S.S.R.}

$\mathrm{T}$ HE following are abstracts of geological topics that appeared in the Soviet journal Priroda during 1962; for convenience they aro arranged under general headings (in italies).

Tungusska 'meteorite'. The so-called Tungusska 'meteorite', which fell in Siberia $\left(60^{\circ} 55^{\prime} \mathrm{N}\right.$., $101^{\circ} 57^{\prime}$ W.) on June 30,1908 , is once more in the news as the results of the 1961 expedition are made known. This expedition remapped the directions of the fallen trees over an area of $2,000 \mathrm{~km}^{2}$, and located and investigated the nature of the so-called 'impact' craters and the composition of spherical granules extracted from the ground. According to the conclusions of V. G. Fesenkov (Priroda, 8, 24; 1962) and K. P. Florensky and I. T. Zotkin (Priroda, 8, $31 ; 1962)$, the impact was not that of a meteorite but of a comet which struck the Earth at a glancing angle and exploded. However, according to N. I. Piavchenko (Priroda, 8, 39; 1962) the observed cavities in the peat bog are not due to impact but to ordinary processes occurring in regions of permafrost.
Meteorite crater in Sayan Range. According to M. V. Voroshilov (Priroda, 3, 107; 1962) an elliptical hollow, rimmed by a 'bulge' and filled by a lake, some $150-400 \mathrm{~m}$ in diameter, situated on the eastern foothills of the Western Sayan Range, represents a meteorite erater. The author suggests that the meteorite responsible for this hollow fell about 150 years ago.

Biogeochemistry. Recent research in biogeochemistry carried out in the U.S.S.R., including the part played by micro-elements in animal nutrition, rational compensatory feeding and biogeochemical provinces and zones, is discussed by V. V. Kovalsky in the June issue of Priroda $(6,3 ; 1962)$.

Arctic regions. A brief review of recent expeditions to the Soviet Arctic has been made by B. A. Kremer (Priroda, 6, 61; 1962), while an up-to-date general account of the geology of the Arctic region is provided by Y. M. Pushcharovsky (Priroda, 4, 35; 1962). The latter article contains a reduced version of a tectonic map of this region compiled by the 